\title{
Krankenkassenanfragen
}

\section{Schluss mit der Willkür}

\author{
Ab Oktober wird es einen einheitlichen Bundesmantelvertrag für Ärzte geben, der für \\ alle Krankenkassenarten gilt. Darauf haben sich KBV und GKV-Spitzenverband nun geeinigt. \\ Ein wichtiger Punkt: Für Krankenkassenanfragen gibt es künftig weniger Formulare.
}

E: s war eine Einigung in letzter Minute: Denn auf die Punkte, die das erste Beschlusspapier zum neuen einheitlichen Bundesmantelvertrag umfasst, hätten sich KBV und GKVSpitzenverband kurz vor einem möglichen Schiedsamtsverfahren geeinigt, erklärt KBV-Pressesprecher Roland Stahl. Was da auf den ersten Blick so unscheinbar wirkt, wie es Stahl beschreibt, habe für die Praxen eine enorme Bedeutung. Und das stimmt in der Tat. Nicht nur, weil der neue Bundesmantelvertrag, auf den sich KBV und GKV-Spitzenverband Mitte Juli geeinigt haben und der ab Oktober in Kraft tritt, nun endlich einheitliche Regeln für alle Krankenkassenarten schafft und die Trennung in „Bundesmantelvertrag Ärzte“ und „Bundesmantelvertrag Ärzte/Ersatzkassen“ überflüssig macht. Es werden auch erstmals feste Regeln für formlose Krankenkassenanfragen geschaffen. Diese sorgten in der Vergangenheit immer wieder für Ärger und überhöhten bürokratischen Aufwand in den Praxen.

\section{Feste Regeln für} "formlose Anfragen"

Jede Krankenkasse konnte dem Arzt einen beliebigen Vordruck zusenden und sich eine Bestimmung des Sozialgesetzbuches oder einer anderen Rechtsvorschrift heraussuchen, nach der die Übermittlung der Information zulässig ist. Die Vergütung für den Mehraufwand des Arztes war damit ebenfalls kaum geregelt. Ab Oktober wird das anders: Dann müssen die Krankenkassen für formlose Anfragen bei Ärzten ein einheitliches Rahmenformular verwenden. Das wird zwar erst noch von den
Partnern des Bundesmantelvertrages entwickelt, aber, so viel steht bereits fest: Aus dem Rahmenformular soll sich auch die Rechtsgrundlage für die Anfrage ergeben. Das soll Rechtssicherheit insbesondere in Bezug auf Schweigepflicht und Datenschutz geben. Außerdem wird der wichtige Punkt der Vergütung geklärt. Zwar kann die KBV hier auch noch keine Größenordnung nennen. Das Formular werde jedoch einen Hinweis auf eine mögliche Vergütung enthalten. Das Formular soll überdies helfen, die lästigen Rückfragen der Krankenkassen beim Arzt zu vermeiden. Damit dies gelingt, wollen die Vertragspartner die Vordrucke regelmäßig überprüfen.

\section{Keine Haftung für ungültige Versichertenkarten}

Mehr Rechtssicherheit gibt es für Ärzte künftig auch bei der elektronischen Gesundheits- beziehungsweise Versichertenkarte. Legt ein Patient eine ungültige Versichertenkarte oder die einer anderen Person vor, kann die Krankenkasse gegenüber dem Arzt keine Schadenersatzansprüche anmelden. Eine Ausnahme bestehe nur insoweit, als der Arzt die unzulässige Verwendung, zum Beispiel aufgrund des Alters, Geschlechts oder des Bildes hätte erkennen können, heißt es in einem Informationsschreiben der $\mathrm{KBV}$ an die KVen. Andersherum kann aber die KV mit dem neuen Bundesmantelvertrag unter Umständen Schadenersatz bei der Krankenkasse einfordern. Sie kann nämlich das Honorar des Arztes, der einen Patienten mit ungültiger Versichertenkarte behandelt hat, der Krankenkasse in Rechnung stellen. Interessant ist dabei: Will die Krankenkas- se diesen Anspruch umgehen, muss sie nachweisen, dass die KV für diesen Versicherten bereits einen Anteil an der Gesamtvergütung erhalten hat. Außerdem darf der Patient die Leistung zwischenzeitlich nicht aus eigener Tasche bezahlt haben.

\section{Stempel im Bonusheft nun doch Pflichtleistung?}

Der neue Bundesmantelvertrag schafft auch Klärung in Sachen Bonushefte für Versicherte. Hier hätten sich KBV und Krankenkassen auf einen Kompromiss geeinigt, erklärt Stahl. Zwar wird ein Passus aufgenommen, wonach das Ausfüllen eines Bonusheftes im Zusammenhang mit der Bestätigung von gesundheitsbewusstem Verhalten eines Versicherten zur vertragsärztlichen Versorgung gehört. Der Arzt muss das Bonusheft jedoch nur dann ohne gesonderte Vergütung ausfüllen, wenn Durchführung und Dokumentation der Leistung im selben Quartal erfolgen.

\section{Anstellung von Ärzten wird erleichtert}

Das für Praxen geltende Verbot der arztgruppenübergreifenden Anstellung von Ärzten, die nur auf Überweisung tätig werden oder überweisungsgebundene Leistungen durchführen, wird aufgehoben. „Das bedeutet, dass sich Ärzte für Laboratoriumsmedizin, Mikrobiologie und Infektionsepidemiologie, Nuklearmedizin, Pathologie, radiologische Diagnostik beziehungsweise Radiologie, Strahlentherapie und Transfusionsmedizin künftig in Praxen anstellen lassen können, die ohne Überweisung in Anspruch genommen werden können", schreibt die KBV. Rebekka Höhl 